\title{
Bony cystic lesion with associated submandibular lymphadenopathy on a background of breast carcinoma: an unexpected case of cervicofacial actinomycosis
}

\author{
Lwazi Sibanda, ${ }^{1}$ Emma Wates (D) , ${ }^{2}$ James Higginson (i) ${ }^{3}$
}

${ }^{1}$ Department of Oral and Maxillofacial Surgery, Worcestershire Acute Hospitals NHS Trust, Worcester, UK 2University of Bristol Medical School, Bristol, UK ${ }^{3}$ Department of Oral and Maxillofacial Surgery, Queen Elizabeth Hospital Birmingham, Edgbaston, UK

\section{Correspondence to}

Emma Wates;

emma.wates@nhs.net

Accepted 4 March 2020

\section{SUMMARY}

Actinomycosis is an uncommon, chronic suppurative granulomatous infection and needs to be considered as a differential diagnosis. A 56-year-old woman with a background of type 2 diabetes mellitus and breast carcinoma was referred to the Oral and Maxillofacial Surgery 2-week wait clinic, regarding a tender sublingual mass and firm erythematous swelling in the right submandibular and submental region. This was slowly progressive and had not responded to oral coamoxiclav. An orthopantomogram showed a well-defined radiolucency and smaller radiolucent lesions throughout the edentulous right body of the mandible. A contrastenhanced CT confirmed a right submandibular abscess communicating with cavitating lesions. The differentials included osteomyelitis, bony metastases, multiple myeloma or other cystic lesions. The patient underwent incision and drainage of the abscess, alongside biopsies, and intravenous co-amoxiclav was given. Microbiology cultures confirmed the presence of Actinomyces israelii and a diagnosis of cervicofacial actinomycosis with mandibular osteomyelitis. The patient was successfully treated with prolonged antibiotics.

\section{BACKGROUND}

Actinomycosis is a rare subacute or chronic suppurative granulomatous infection caused by bacteria from the Actinomyces genus. These gram-positive filamentous bacteria are opportunistic pathogens: usually commensal in the upper aerodigestive tract, and becoming invasive following disruption of the

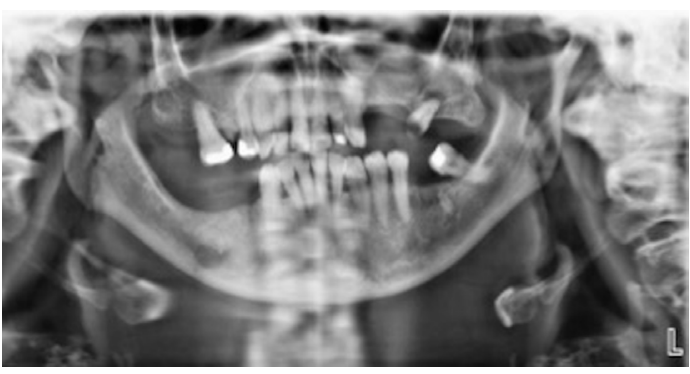

Figure 1 Preoperative orthopantomogram showing well-defined radiolucency in the right body of the mandible, as well as other smaller radiolucent lesions throughout the middle of the mandible.

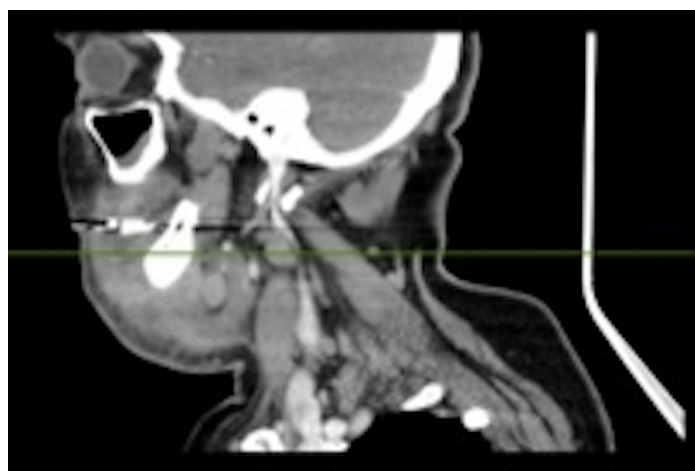

Figure 2 Sagittal view of the contrast-enhanced $\mathrm{CT}$ of the neck showing a cystic lesion in the right submandibular area extending to a small cavitating lesion in the right mandible, with evidence of extensive swelling of right mylohyoid muscle and floor of the mouth. Enlarged lymph nodes in right submandibular and submental area are seen.

mucosal barrier due to trauma, surgical procedures or the presence of foreign bodies. ${ }^{1}$ Actinomycosis can affect cervicofacial, thoracic, abdominal and pelvic regions, with cervicofacial infection, usually of the mandible, being the most common. Actinomyces israelii is the most frequently isolated causative species. $^{12}$ The Actinomyces spp are found in a variety of polymicrobial infections, particularly associated with the head and neck.

On examination, actinomycosis commonly presents as a woody-hard swelling. ${ }^{3}{ }^{4}$ The subacute nature of actinomycosis, along with its rarity and the length of time required to make a formal microbiological diagnosis, mean that it is commonly mistaken for malignancy, granulomatous disease or a fungal infection.

We present a case in which diagnosis was delayed because of a mistaken diagnosis of malignancy.

\section{CASE PRESENTATION}

A 56-year-old woman was referred by her general medical practitioner (GMP) to the Oral and Maxillofacial Surgery (OMFS) 2-week wait clinic for suspected head and neck cancer, for the evaluation of a tender sublingual mass associated with a swelling in the right submandibular and submental regions of the neck. This had been slowly 


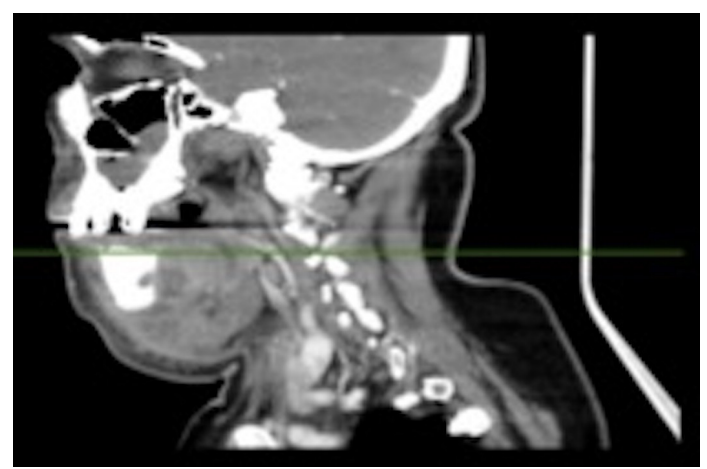

Figure 3 Sagittal view of the contrast-enhanced CT of the neck showing a cystic lesion in the right submandibular area extending to a small cavitating lesion in the right mandible, with evidence of extensive swelling of right mylohyoid muscle and floor of the mouth. Enlarged lymph nodes in right submandibular and submental area are seen.

progressive for over a month and had not responded to a course of oral co-amoxiclav prescribed by her GMP.

The patient had a background of type 2 diabetes mellitus, asthma, psoriasis, hypertension, depression and breast carcinoma 10 years ago. Her breast cancer was previously treated with a right mastectomy and axillary node clearance, followed by radiotherapy, then adjuvant hormonal therapy, which was ongoing at the time of presentation. She was a non-smoker and drank alcohol within the recommended limits.

On examination, there was a firm tense erythematous swelling in the right submandibular and submental regions. She had no significant restriction of mouth opening. Intraorally, there was a firm area palpable in the right side of the floor of mouth adjacent to the mandible. However, there were no obvious mucosal lesions.

A working diagnosis of locoregionally advanced head and neck squamous cell carcinoma (HNSCC) was made, and the patient underwent investigations accordingly.

\section{INVESTIGATIONS}

An orthopantomogram (OPG) (figure 1) showed a well-defined, non-corticated radiolucency in the right body of the mandible, as well as several other smaller radiolucent lesions throughout the mandible. As per recommended investigations for suspected malignancy, a contrast-enhanced CT scan of the neck and mandible was taken (figures 2-5), alongside histopathological biopsies to exclude malignancies, including metastases. This confirmed a $20 \times 18 \mathrm{~mm}$ cystic lesion in the right submandibular triangle, communicating with a small cavitating lesion in the right mandible. It also showed enlarged right submandibular and submental lymph nodes (measuring up to $15 \mathrm{~mm}$ and up to $8 \mathrm{~mm}$, respectively).

Blood tests were performed, and the full blood count was unremarkable, with a white cell count of $8.3 \times 10^{9} / \mathrm{L}$ and a neutrophil count of $6.6 \times 10^{9} / \mathrm{L}$. Urea and electrolyte tests showed a borderline hypokalaemia of potassium of $3.2 \mathrm{mmol} / \mathrm{L}$, but were otherwise unremarkable. Inflammatory markers showed an elevated erythrocyte sedimentation rate of $120 \mathrm{~mm} /$ hour and C-reactive protein of $97 \mathrm{mg} / \mathrm{L}$. Bone markers included an adjusted calcium of $2.43 \mathrm{mmol} / \mathrm{L}$ and alkaline phosphatase of $135 \mathrm{iu} / \mathrm{L}$. The $\mathrm{HbA} 1 \mathrm{c}$ was $86 \mathrm{mmol} / \mathrm{mol}$. All fractions were raised in protein electrophoresis with no evidence of paraproteins. The urinary Bence Jones protein test was negative.

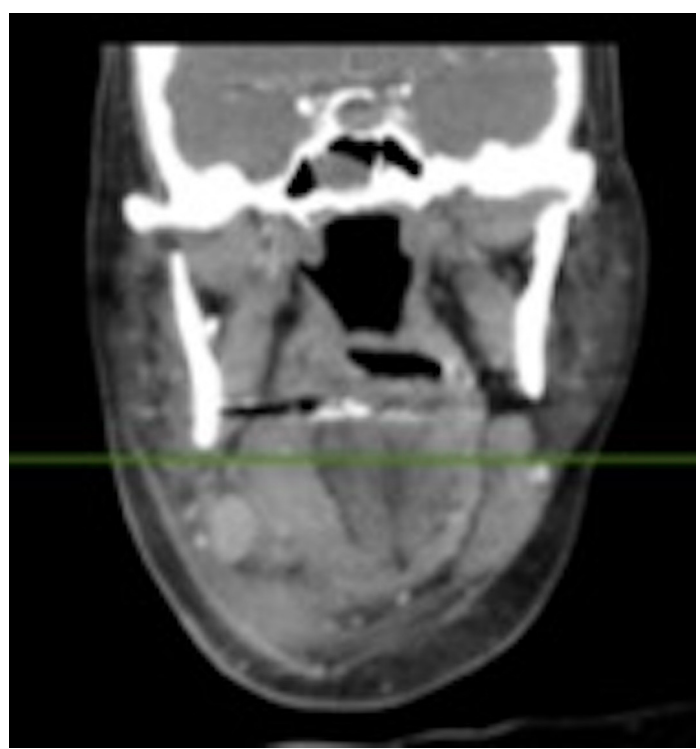

Figure 4 Coronal view of the contrast-enhanced CT of the neck showing a cystic lesion in the right submandibular area extending to a small cavitating lesion in the right mandible, with evidence of extensive swelling of right mylohyoid muscle and floor of the mouth. Enlarged lymph nodes in right submandibular and submental area are seen.

\section{DIFFERENTIAL DIAGNOSIS}

The main differential diagnoses were locoregionally advanced HNSCC, bony metastases from breast carcinoma, multiple myeloma, mandibular osteomyelitis, benign odontogenic mandibular cysts (including ameloblastoma and odontogenic keratocyst) and osteomyelitis of the mandible.

Abscesses and fistulae due to acute infection with pyogenic bacteria were thought to be unlikely because of the gradual onset of the swelling, the patient's good general health and the absence

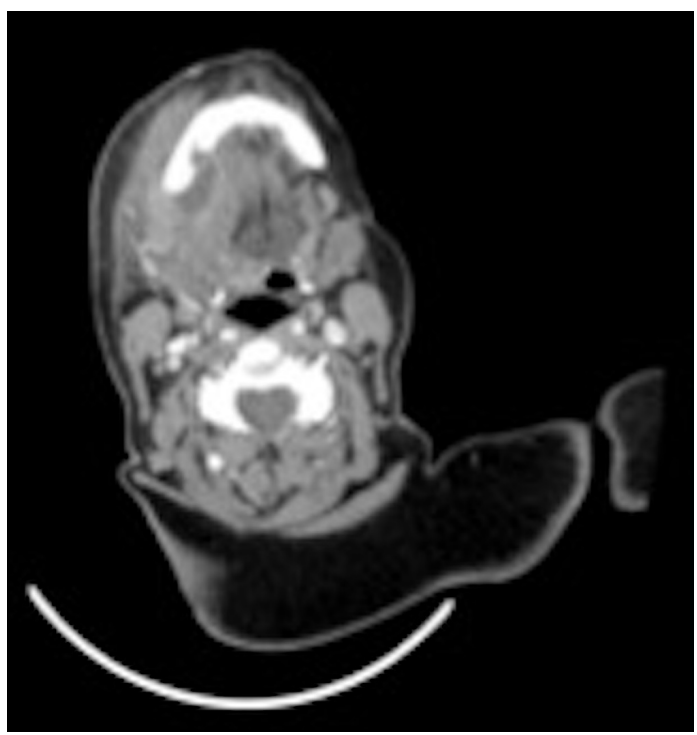

Figure 5 Axial view of the contrast-enhanced CT of the neck showing a cystic lesion in the right submandibular area extending to a small cavitating lesion in the right mandible with evidence of extensive swelling of right mylohyoid muscle and floor of the mouth. Enlarged lymph nodes in right submandibular and submental area are seen. There is a possibility of associated mandibular osteomyelitis. 
of leucocytosis. Due to the rarity of cervicofacial actinomycosis, it was not included in the initial list of differentials.

The diagnosis of cervicofacial actinomycosis was not able to be made until the pus swab collected from her initial outpatient appointment confirmed the moderate growth of $A$. israelii. The absence of Bence Jones proteins from the urine sample at this appointment ruled out multiple myeloma, and histology of the specimen showed reactive bone and chronically inflamed stroma, suggestive of chronic osteomyelitis with no evidence of malignancy or of a plasma cell infiltrate.

\section{TREATMENT}

The patient was prescribed intravenous co-amoxiclav and dexamethasone and underwent an examination under anaesthesia. The submental and submasseteric spaces were explored by blunt dissection, with moderate quantities of pus released and biopsies, including bone curettage samples, were sent for histopathology.

\section{OUTCOME AND FOLLOW-UP}

The patient recovered well and was discharged 2 days after her operation on oral co-amoxiclav.

The patient was reviewed, as an outpatient, 5 days postoperatively, and a reduction in the indurated swelling was noted with minimal drainage. She was prescribed a 4-week course of doxycycline to commence after completing her course of antibiotics. At a further outpatient review 2 weeks later, she had further reduction in swelling, and a sinus in the right submental region, with tenderness to palpation in the right buccal sulcus and a pale pink discharge. She was then prescribed a 3-week course of phenoxymethylpenicillin in light of the microbiology report of the presence of the Actinomyces species.

Further to this, at her 3-week postoperative review with wound closure over her right mandible, and after discussion on the microbiology report, the patient was prescribed a 3-month course of oral amoxicillin. On her 3-month review, the patient was asymptomatic. Clinically, the patient had no palpable swellings or trismus and intraorally no swellings were found. An MRI taken revealed no significant abnormalities and her OPG showed improvement of her radiolucency on her right mandible.

\section{DISCUSSION}

This case highlights to individuals working in OMFS settings that actinomycosis is an important differential diagnosis for patients presenting with submental and submasseteric masses.

It is difficult to diagnose actinomycosis based solely on clinical history, examination and radiological investigations. There are many differential diagnoses for a submandibular and submental swellings, including both acute and chronic diagnoses. Some of these include bony cysts, metastases from the breast carcinoma, infectious causes, including tuberculosis, and acute and chronic osteomyelitis. ${ }^{5}$ Therefore, investigations must be carried out promptly to establish the definitive diagnosis.

This case highlights the importance of sending off the pus swabs, even in the absence of acute suppurative infection, as the radiological, biochemistry and haematology investigations were not indicative of actinomycosis. In this case, the definitive diagnosis was made based on the microbiology report, before this actinomycosis was not considered due to its rarity in the UK, $0.0006 \%$ of hospital consultations in the UK presented with actinomycosis, ${ }^{2}$ with approximately $55 \%$ of these being in the orocervicofacial region. ${ }^{6}$ It is necessary to make the microbiology laboratory aware whether an antimicrobial treatment regime has commenced before sample collection, as the results of culture in this context could lead to false negatives.

Plain radiographs were useful for ruling out an acute odontogenic cause for the disease and also have a role assessing the progression of disease by comparing changes seen on follow-up films with the initial radiograph. ${ }^{7}$ Plain radiographs are not definitive, however, and contrast-enhanced CT scans or MRI scans should be used to clarify the significance of ambiguous plain radiographic findings, such as equivocal lytic lesions. ${ }^{8}$ Contrast-enhanced CT scans are quick, accurate and demonstrate the extent of infection, distortion of anatomical structures or vascular compromise. Both contrast-enhanced CT scans and MRI scans may yield inconclusive findings, but are useful in planning the surgical treatment and also in determining resolution. ${ }^{8}$

The correct antibiotic regime is of crucial importance, as Actinomyces spp are not sensitive to a number of commonly prescribed antibiotics, including metronidazole, aminoglycosides, aztreonam, co-trimoxazole, penicillinase-resistant penicillins and fluoroquinolones (ciprofloxacin and moxifloxacin). However, the Actinomyces spp are usually susceptible to betalactam antibiotics, especially penicillin $G$ or amoxicillin. ${ }^{8}$ This reinforces the importance of establishing the correct diagnosis promptly, so that the correct antibiotic regime can be commenced early to avoid further complications of inadequately treated actinomycosis, including bony destruction, abscesses, multiple draining fistulas and the need for aggressive debridement, potentially requiring free flap reconstruction. ${ }^{3}$

Historically, actinomycosis was treated with long-term courses (usually 4-6 weeks) of intravenous antibiotics followed by oral doses. However, short course regimens have been proved to be successful. ${ }^{9}$ This is necessary in combination with adequate drainage where an abscess is present. ${ }^{7910}$

This presented case highlights the importance of the OMFS team considering actinomycosis as a differential: in this instance, the diagnosis was not made initially and, therefore, long-term treatment was delayed. Hence, we need to be aware of the signs and symptoms suggesting actinomycosis to consider it as a key differential.

\section{Learning points}

- Cervicofacial actinomycosis should be included in the differential diagnosis of a relatively homogeneously enhancing soft tissue mass associated with substantial inflammatory reaction and infiltration of the cervicofacial area.

- This case highlights the importance of sending off the pus swabs as the radiological, biochemistry and haematology investigations may not be indicative of actinomycosis.

- Osteomyelitis is a complication of actinomycosis if left untreated.

- The most appropriate antibiotics are penicillin G or amoxicillin, and success has been reported with short but intensive courses, though advice should always be sought from local microbiology services.

Twitter James Higginson @jimhigginson

Contributors LS wrote the paper and conducted the literature review and EW and $\mathrm{JH}$ revised the draft paper.

Funding The authors have not declared a specific grant for this research from any funding agency in the public, commercial or not-for-profit sectors.

Competing interests None declared.

Patient consent for publication Obtained. 
Rare disease

Provenance and peer review Not commissioned; externally peer reviewed.

\section{ORCID iDs}

Emma Wates http://orcid.org/0000-0001-5531-1891

James Higginson http://orcid.org/0000-0002-6913-1557

\section{REFERENCES}

1 Nagler R, Peled M, Laufer D. Cervicofacial actinomycosis: a diagnostic challenge. Oral Surg Oral Med Oral Pathol Oral Radiol Endod 1997;83:652-6.

2 Wong VK, Turmezei TD, Weston VC. Actinomycosis. BMJ 2011;343:d6099.

3 Shen JY, Futran ND, Sardesai MG. Craniofacial Actinomyces osteomyelitis evolving from sinusitis. Radiol Case Rep 2018;13:104-7.

4 Belmont MJ, Behar PM, Wax MK. Atypical presentations of actinomycosis. Head Neck 1999;21:264-8
5 Volante M, Contucci AM, Fantoni M, et al. Cervicofacial actinomycosis: still a difficult differential diagnosis. Acta Otorhinolaryngol Ital 2005;25:116-9.

6 Moghimi M, Salentijn E, Debets-Ossenkop Y, et al. Treatment of cervicofacial actinomycosis: a report of 19 cases and review of literature. Med Oral Patol Oral Cir Bucal 2013;18:e627-32.

7 Oostman 0, Smego RA. Cervicofacial actinomycosis: diagnosis and management. Curr Infect Dis Rep 2005;7:170-4.

8 Park JK, Lee HK, Ha HK, et al. Cervicofacial actinomycosis: CT and MR imaging findings in seven patients. AJNR Am J Neuroradiol 2003;24:331-5.

9 Shah KM, Karagir A, Kanitkar S, et al. An atypical form of cervicofacial actinomycosis treated with short but intensive antibiotic regimen. BMJ Case Rep 2013;2013:bcr2013008733.

10 Valour F, Sénéchal A, Dupieux C, et al. Actinomycosis: etiology, treatment, and management. Infect Drug Resist 2014;7:183-97.

Copyright 2020 BMJ Publishing Group. All rights reserved. For permission to reuse any of this content visit

https://www.bmj.com/company/products-services/rights-and-licensing/permissions/

BMJ Case Report Fellows may re-use this article for personal use and teaching without any further permission.

Become a Fellow of BMJ Case Reports today and you can:

- Submit as many cases as you like

- Enjoy fast sympathetic peer review and rapid publication of accepted articles

- Access all the published articles

- Re-use any of the published material for personal use and teaching without further permission

\section{Customer Service}

If you have any further queries about your subscription, please contact our customer services team on +44 (0) 2071111105 or via email at support@bmj.com.

Visit casereports.bmj.com for more articles like this and to become a Fellow 